\title{
Implementation of Microstructural Material Phenomena in Macro Scale Simulations of Forming Processes
}

\author{
J. Huétink \\ University of Twente, Faculty of Engineering Technology \\ and Netherlands Institute for Metals Research (NIMR) \\ P.O.Box 217, 7500 AE Enschede, the Netherlands \\ email: j.huetink@ctw.utwente.nl
}

\begin{abstract}
The paper deals with problems related to full/macro scale simulations of industrial forming processes. Large-scale numerical simulations and virtual modeling are replacing prototypes in order to reduce costs and time. This requires accurate and reliable predictions. To satisfy these requirements, sophisticated material models including microstructural phenomena as phase transitions, aging, and recrystallization have to be considered on macro scale level simulation. Solution strategies are discussed and some examples are given of complex thermo- mechanically coupled forming simulations.
\end{abstract}

\section{INTRODUCTION}

Developing prototypes and redesign based on tryout with these prototypes is time consuming and expensive. Prototypes are replaced more and more by virtual modeling using numerical simulations in order to reduce developing costs and time. Prediction based on these simulations should be accurate and reliable and the computation time should be acceptable. To simulate forming processes this requires sophisticated material models including micro structural phenomena as phase transitions, aging, and recrystallization. In materials science numerical modeling is becoming a popular tool to gain insight in the physical properties and micro-structural changes that occur during processing. Observed micro structural phenomena are cast into mathematical models and implemented in FEM codes $[1,2,3]$. In these micro level FEM models single crystals, aggregates or dislocations are modeled by several elements. These numerical micro scale models cannot directly be applied on macro-scale. A Multi Level Finite Element Method (MLFEM) procedure is being developed to bridge the gap between micro and macro scale models [4,5]. Via homogenization steps using Representative Volume Elements (RVE), constitutive relations are generated on integration point level of the macro model. For small two-dimensional academic problems this approach shows promising results. However the computation time of these kind of methods is still too high for application in full scale three dimensional forming process simulations. As long as these MLFEM procedures are too much computer time consuming, approximations have to be used in which micro-structural phenomena are translated into averaged continuum constitutive relations. In the following sections some examples will be shown of macro-scale thermo mechanically coupled simulations using constitutive equations based on physically observed micro structural evolution. The last section presents an algorithm to speed up complex (thermomechanically coupled sheet forming processes.

\section{WARM FORMING OF ALUMINIUM SHEET}

The formability of $\mathrm{Al}-\mathrm{Mg}$ sheet can be improved considerably, by increasing the temperature $[6,7,8]$. At elevated temperatures, the mechanical response of the material becomes strain rate dependent. By heating the sheet in areas with large shear strains, but cooling it on places where the risk of necking is high, the limiting drawing ratio can be increased to values above 2.5 . To accurately simulate warm forming of aluminium sheet, a material model is required that incorporates the temperature and strain-rate dependency. In this section simulations are presented of the deep drawing of a cylindrical cup, using shell elements which are extended with temperature degrees of freedom. It is demonstrated that the familiar quadratic Hill yield 
function is not capable of describing the plastic deformation of aluminium. Hence, the Vegter Yield criterion [13] is used. With this criterion it is possible to approximate a yield criterion obtained from crystal plasticity models rather accurately. Currently available constitutive equations based on crystal plasticity [1] require to much computer time for application in full scale simulations. By using the Vegter criterion the computer time can be reduced considerably. A disadvantage is that only the initial texture can be taken into account and not texture evolution during forming. More details can be found in $[6,7,8]$. Hardening can be described succesfully with the physically based material model for temperatures up to $200{ }^{\circ} \mathrm{C}$. This model considers the evolution of the dislocation density based on storage and dynamic recovery processes. At higher temperatures and very low strain rates, the flow curve deviates significantly from the modeland more 'state-variables' then only one dislocation density is needed.

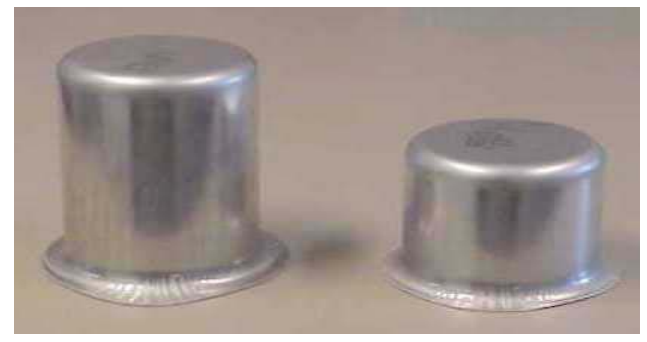

FIGURE 1. Warm forming of aliminium sheet. Deep drawing of a cup at $250{ }^{\circ} \mathrm{C}$ (left) and $20{ }^{\circ} \mathrm{C}$ (right).

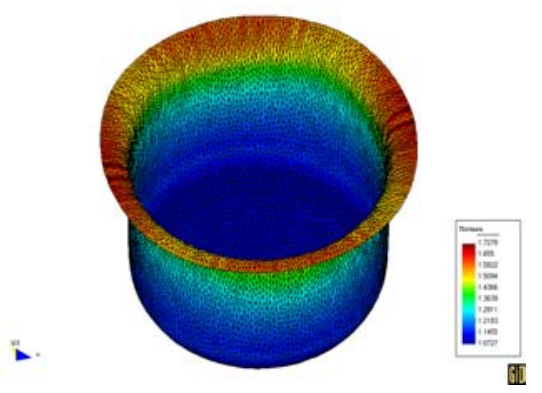

FIGURE 2. Simulation of warm deep drawing of an aluminum cup [7].

\section{FORMING OF CORROSION RESISTANT METASTABLE STEEL SHEET}

A model to describe the constitutive behaviour of Sandvik corrosion-resistant steel Nanoflex ${ }^{\circledR}$ [15] during metal forming and hardening is developed for application in forming simulations. The material is metastable, which causes strain-induced transformation to take place during metal forming. Depending on the annealing conditions, the material will also transform isothermally $[16,17]$ (as the opposite to athermal martensite). This transformation can also take place immediately after forming, as a result of the residual stresses present in the material. The martensite phase of this material can be aged [18]. In this section results of the various measurements on Sandvik Nanoflex are shown. The measurements mainly involved tensile tests and upsetting tests, in which both isothermal transformation and straininduced transformation were measured inductively [19]. The hardening of the material and the increase in hardness during ageing was also examined. Based on experimental results and literature a constitutive model is developed [9]. The model describes isothermal, stress-assisted transformation, strain-induced transformation, work hardening and ageing. The model has been set up in such a way that it can be simply implemented in a FEM code.

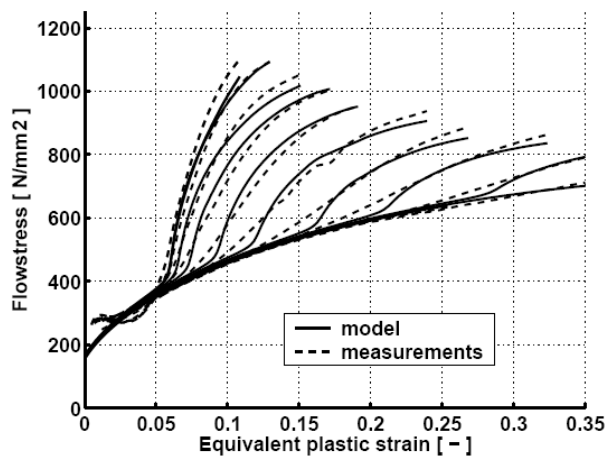

FIGURE 3. Experimental and predicted stress strain curves. The most left lines correspond to a tempareture of $223 \mathrm{~K}$ whereas the most right lines correspond to $423 \mathrm{~K}$. Temperature steps are $20 \mathrm{~K}$

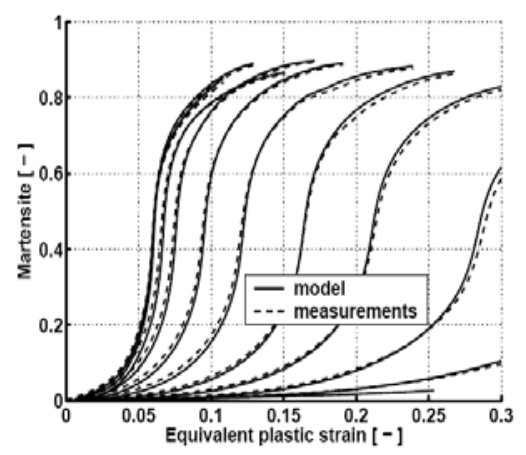

FIGURE 4. Phase transition from austenite to martensite during tensile test corresponding to figure 3 . 
The measured and predicted stress strain curves and corresponding phase transitions during the tensile tests are given in figure 3 and figure 4 . The numerical results are in good agreement with the experiments. For a mathematical formulation and other data the reader is referred to [9].

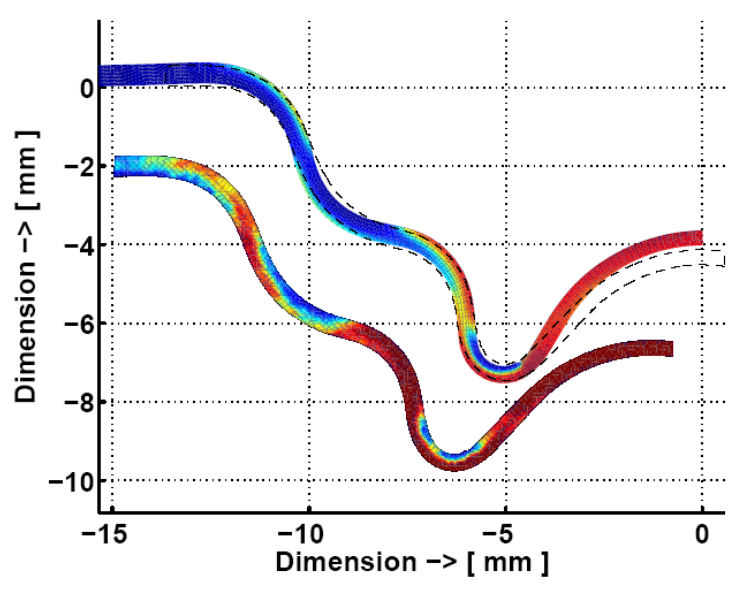

FIGURE 5. Prediction of the fraction of martensite formed based on thermodynamic calculations for the various phases of Sandvik NanoFlex ${ }^{\circledR}$ : calculated (top contour) vs. experiment (bottom contour) and the dotted line represents the experimental final specimen shape after deformation (red: $100 \%$ martensite; blue: no martensite).

The model in implemented in a dedicated FEM code. Figure 5 shows a result of a test simulation. The deviation of the desired shape is mainly attributed to the inhomogeneous transformation and the corresponding difference in density of the austenite and martensite phase respectively. More details concerning the simulation and the material model are given in [20].

\section{EFFICIENT IMPLICIT FINITE ELEMENT ANALYSIS OF SHEET FORMING PROCESSES.}

The computation time for implicit finnite element analyses tends to increase disproportionally with increasing problem size. This is due to the repeated solution of linear sets of equations, if direct solvers are used. By using iterative linear equation solvers the total analysis time can be reduced for large systems. For plate or shell element models, however, the condition of the matrix is so ill that iterative solvers do not reach the huge time-savings that are realized in case of with solid element models. By introducing inertial effects into the implicit finite element code the condition number can be improved and iterative solvers perform much better $[10,11,12]$. An additional advantage is that the inertial effects stabilize the Newton-Raphson

iterations. This also applies to quasi-static processes, for which the inertial effects finally do not affect the results. The presented method can readily be implemented in existing implicit finite element codes. Industrial size deep drawing simulations are executed to investigate the performance of the recommended strategy. It is concluded that the computation time is decreased by a factor of 5 to 10 . As an example the result for a simulation of the NUMISHEET 2002 Benschmark 3 is presented. Figure 6 shows the final mesh after the first forming step.

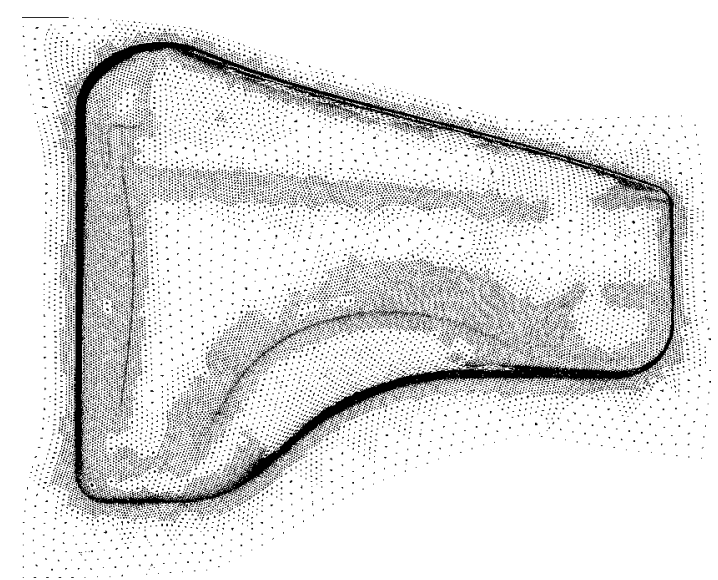

FIGURE 6. Numisheet 2002 Benchmark 3 prediction using an iterative solver and including inertia

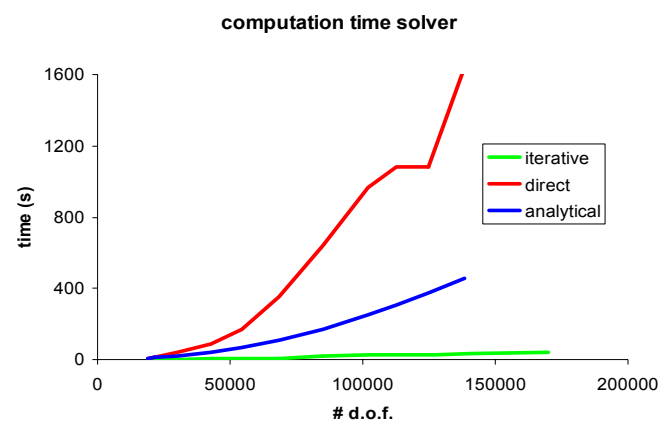

FIGURE 7. Comparison of the solver time for a direct solver and the iterative solver with inertia, as a function of the degrees of freedom.

Figure 7 shows a comparison between the calculation time using a direct solver and an iterative solver including inertia. The number of degrees of freedom is increased by an automatic mesh refinement procedure. Similar as to explicit calculations, the calculation time can be reduced by artificial mass scaling at the cost of accuracy. The iterative solver procedure is also applied to thermo-mechanically coupled problems. The result 
from the warm forming simulation shown in Figure 2 is obtained by applying this iterative solver.

\section{ACKNOWLEDGMENTS}

The work presented is this paper is the result of a long time cooperation between the Mechanics of Forming Processes Group at the University of Twente, the Netherlands Institute for Metals Research (NIMR), Philips and Corus Group. In this papers some high lights are collected which are extracted from report and paper written within that framework.

\section{REFERENCES}

1. Reis, A., Santos, A.D., Ferreira Duarte J., Rocha A.B., Say-Yi Li, Hofelin, E., Van Bael A., Van Houtte, P., Teodosiu, C.: Experimental validation of a new plasticity model of texture and strain-induced anisotropy. The 4th International Esaform Confeence, 2002, pp. 433-436.

2. Habraken, A-M.: Contribution to constitutive laws of metals: micro-macro and damage models. Thesis (2001).

3. V. S. Deshpande, E. Van der Giessen and A. Needleman, "Discrete Dislocation Plasticity Modeling of Short Cracks in Single Crystals," Acta Materalia, v. 51, p. 115, 2003.

4. Smit R.J.M., Brekelmans W.A.M., and Meijer H.E.H. (1998) Comput. Methods Appl. Mech. Engrg., 115, 181192.

5. Kouznetsova, V. et al (2001), Comp Mech 27, 37-48

6. Van den Boogaard, A. H., Bolt, P. J., and Werkhoven, R. J., International Journal of Forming Processes, 4, 361375 (2001).

7. Van den Boogaard, A. H., Thermally enhanced forming of aluminium sheet-Modelling and experiments, Ph.D. thesis, University of Twente (2002).

8. A.H. van den Boogaard and J. Huétink, Modelling of aluminium sheet forming at elevated temperatures, Numiform 2004.

9. J.Post, K. Datta and J. Huétink, Constitutive behaviour of the metastable stainless steel: Sandvik Nanoflex ${ }^{\circledR}$,. Numiform 2004.

10. T. Meinders, A.H. van den Boogaard, J. Huétink, An algorithm to make your implicit code competitive with an explicit code for large scale problems, Numisheet 2002.
11. T. Meinders, A.H. van den Boogaard, J.Huétink, Improvement of implicit finite element code performance in deep drawing simulations by dynamics contributions, J. of Mat. Proc. Tech.134,413-420.(2003)

12. A.H. van den Boogaard, T.Meinders, J.Huétink, Efficient implicit finite element analysis of sheet forming processes, I.J.N.M.E., vol.56, Issue 8, 2003

13. Vegter, H., and Van den Boogaard, A. H., International Journal of Plasticity (2004), submitted.

14. Bergström, Y., Materials Science and Engineering, 5, 193-200 (1969).

15. M. Holmquist, J.-O. Nilsson, and A.H. Hultin Stigenberg. Isothermal formation of martensite in a $12 \mathrm{cr}-$ 9ni- 4mo maraging stainless steel. Scripta Metallurgia et Materialia, January 1995.

16. G.B. Olson and Morris Cohen. Kinetics of strain-induced martensic nucleation. Metallurgical Transactions A, 6A:791-795, April 1975.

17. I. Tamura and C. M. Wayman, "Martensitic Transformations and Mechanical Effects" in the book "Martensite", ed. G. B. Olson and W. S. Owen, ASM International, 1992, ISBN: 0-87170-434-X.

18. J.-O. Nilsson, A.H. Hultin Stigenberg, and P. Liu. Isothermal formation of quasicrystalline precipitates and their effect on strength in a 12cr-9ni-4mo maraging stainless steel. Metallurgical and Materials Transactions A, 25A:2225-2233, October 1994.

19. H. Nolles ea. Inductive measurements of the stress assisted and strain induced martensite transformations of sandvik maraging steel 1rk91 before, during and after metal forming. ICOMAT 2002, (2003).

20. R.M.J.Voncken, O. van der Sluis, J. Post and J. Huétink FE calculations on a three stage metal forming process of Sandvik Nanoflex ${ }^{\circledR}$, Numiform 2004. 